\title{
Diagnostic accuracy of nasal endoscopy and contrast magnetic resonance imaging in COVID-19 associated mucormycosis (CAM)
}

\author{
Nadeem Shaikh ${ }^{1}$, Neha Shakrawal ${ }^{1}$, Mahendra Chouhan $^{1}$, and Bharti Solanki ${ }^{1}$ \\ ${ }^{1}$ Dr Sampurnanand Medical College
}

October 19, 2021

\begin{abstract}
Key Points 1. COVID-19 associated mucormycosis (CAM) is a life-threatening fungal disease that has become an epidemic and notifiable disease after the second wave of COVID-19 in India 2. The target group for CAM includes severely immunocompromised individuals either by COVID-19, or corticosteroid therapy, or uncontrolled glycemic index. 3. With an intention to find out the sensitivity and specificity of diagnostic nasal endoscopy (DNE) and contrast-enhanced magnetic resonance imaging (CEMRI), one hundred two consecutive patients of target group with clinical features of CAM were enrolled in the study. 4. The sensitivity and specificity of DNE as compared to $\mathrm{KOH}$ mount were $73.4 \%$ and $94.7 \%$ respectively. The sensitivity and specificity of CEMRI as compared to $\mathrm{KOH}$ mount were $87.5 \%$ and $50 \%$ respectively. 5. CEMRI misses fewer cases than DNE. In cases of normal endoscopy in the target group, imaging and biopsy should be advised to reduce false-negative results. Nasal endoscopy, biopsy, and imaging can reliably detect the majority of cases of CAM.
\end{abstract}

\section{Key Points}

1. COVID-19 associated mucormycosis (CAM) is a life-threatening fungal disease that has become an epidemic and notifiable disease after the second wave of COVID-19 in India

2. The target group for CAM includes severely immunocompromised individuals either by COVID-19, or corticosteroid therapy, or uncontrolled glycemic index.

3. With an intention to find out the sensitivity and specificity of diagnostic nasal endoscopy (DNE) and contrast-enhanced magnetic resonance imaging (CEMRI), one hundred two consecutive patients of target group with clinical features of CAM were enrolled in the study.

4. The sensitivity and specificity of DNE as compared to KOH mount were $73.4 \%$ and $94.7 \%$ respectively. The sensitivity and specificity of CEMRI as compared to $\mathrm{KOH}$ mount were $87.5 \%$ and $50 \%$ respectively.

5. CEMRI misses fewer cases than DNE. In cases of normal endoscopy in the target group, imaging and biopsy should be advised to reduce false-negative results. Nasal endoscopy, biopsy, and imaging can reliably detect the majority of cases of CAM.

\section{Introduction}

COVID-19 associated mucormycosis (CAM) is the result of the severe immunosuppression caused by COVID19 and its management ${ }^{1}$. The first series of CAM was published in February $2021^{2}$, since then there is an upsurge of these cases. The target group for CAM includes patients with a history of COVID-19, corticosteroid therapy, and uncontrolled blood sugars. These patients with alarming signs and symptoms should be considered for comprehensive evaluation ${ }^{3}$. The nasal cavity is the first point of contact and the fungus harboring the nasal cavity makes DNE an important tool of diagnosis. But changing trends and varied presentation of the disease in this epidemic include patients with minimal signs instead of established red flag 
symptoms. DNE in such cases is normal. The early stages of CAM need not have necrosis, discoloration, or blackening in the nasal cavity (Figure 1-a,b,c,d). The limitation of endoscopy is in evaluation of the disease beyond the confinements of the nasal cavity. Then comes the role of imaging and biopsy in making an early and accurate diagnosis ${ }^{4}$.

This study was designed to determine the sensitivity and diagnostic accuracy of endoscopy and imaging in comparison to potassium hydroxide calcofluor white mount $\mathrm{KOH}$ mount and correlating them. This will help to formulate an ideal diagnostic strategy in suspected cases of invasive fungal sinusitis.

\section{Materials and Methods}

One hundred two patients of target group with clinical features of CAM presenting to the outpatient Department of Otorhinolaryngology were serially recruited and subjected to DNE by an experienced Otorhinolaryngologist, and a tissue sample for $\mathrm{KOH}$ mount was sent from representative areas. Informed consent was obtained from all individual participants included in the study and ethical clearance was acquired from the institutional ethical committee.

We excluded cases of invasive fungal sinusitis which were diagnosed elsewhere or presented with recurrence or residual diseases. Nasal endoscopy and biopsy for KOH mount were sent for all 102 patients but CEMRI of paranasal sinuses, orbit \& brain could be done in only 60 patients due to financial constraints. Nasal endoscopy was performed in the operation theater using a zero degree rigid endoscope directly and then after decongestion and local anesthesia. Bilateral evaluation was done for all patients to compare both sides. The discoloration, blackening, crusting, or necrotic areas over turbinates, septum, and mucosa were considered as positive findings (Figure 1-e,f). Punch biopsies from the affected tissues were taken. For those with normal DNE, biopsy sample was taken from the middle turbinate of the side the symptoms. The tissue samples were prepared and the $\mathrm{KOH}$ mounts were examined for the presence of fungal elements by experienced microbiologists. The morphological findings including broad aseptate hyphae was considered as evidence of the presence of zygomycosis even though invasive fungal sinusitis cannot be diagnosed solely based on the presence of fungal elements on $\mathrm{KOH}$ mount. Non-enhancement, thin enhancement, heterogenous enhancement, and enhancement with abnormal findings on CEMRI were considered as positive findings (Figure 2).

\section{Results}

\section{Demography}

Out of 102 study population, 69(67.64\%) were male and 33(32.35\%) were female. The age range was 23-77 years with a mean of 51 years with a standard deviation of 12.31 . Most of the patients $(61.76 \%)$ were in the age group [?] 50 years.

Nasal endoscopy of 102 subjects

Out of the study population, $49(48 \%)$ showed positive findings on nasal endoscopy. The most common presentation observed in $92 \%$ of cases was blackish discoloration of turbinate mucosa. Bilateral involvement was seen in 12 patients (24.48\%). Other early signs were discolored or pale mucosa. The endoscopy findings and prevalence of involvement of nasal structures are given in Table 1.

Out of the 49 (48\%) who had positive nasal endoscopic findings, 47 showed broad aseptate hyphae on $\mathrm{KOH}$ mount. Out of $53(52 \%)$ with normal DNE findings, $17(32 \%)$ showed broad aseptate hyphae on the KOH mount. On DNE, we picked up $48 \%$ of cases but $16.7 \%$ were missed due to no striking findings in DNE. The sensitivity and specificity of DNE as compared to $\mathrm{KOH}$ mount were $73.4 \%$ and $94.7 \%$ respectively. The positive predictive value, negative predictive value, and accuracy were $95.5 \%, 67.9 \%$, and $81.37 \%$ respectively.

Imaging evaluation of 60 subjects

Out of 60 patients who underwent CEMRI, the most commonly involved sinus was maxillary sinus as observed in $90 \%$ of the cases. The MRI findings and structures involved are described in Table 1. Bilateral involvement 
was seen in 28 patients (46.7\%). Out of total, 51 showed positive findings in the form of non-enhancement, thin enhancement, or heterogenous enhancement. Out of those 51, 49 were positive for $\mathrm{KOH}$ mount. Out of nine CEMRI with negative findings, seven were positive for $\mathrm{KOH}$ mount. The sensitivity and specificity of CEMRI as compared to $\mathrm{KOH}$ mount were $87.5 \%$ and $50 \%$ respectively. The positive predictive value, negative predictive value, and accuracy were $96.07 \%, 22.22 \%$, and $85 \%$ respectively.

Correlation of diagnostic nasal endoscopy and CEMRI $(\mathrm{n}=60)$

This was done in 60 patients because all three diagnostic tests i.e. endoscopy, biopsy and imaging were done in them. As shown in Table 2, out of a total of 60 patients, $40(66.7 \%)$ had abnormal endoscopic findings while 51 (85\%) patients showed positive CEMRI findings. Out of 20 patients having normal endoscopy, 13 patients had abnormal CEMRI. But two patients who were CEMRI negative had endoscopic features for CAM. Seven patients were both endoscopy and CEMRI negative for CAM indicating no disease. Considering imaging as a superior diagnostic tool for the diagnosis of CAM, the various parameters for endoscopy were calculated. The sensitivity of DNE was $74.50 \%$, specificity was $77.77 \%$, positive predictive value was $95 \%$ and negative predictive value was $35 \%$. Diagnostic accuracy came out to be $75 \%$. Most of the endoscopy positive patients of CAM were CEMRI positive. Agreement between DNE and CEMRI in diagnosis of CAM was good with a kappa coefficient of 0.370 .

\section{Discussion}

COVID-19 has recently been responsible for a drastic surge in the cases of CAM. We described the DNE findings of 102 patients in the target group who underwent $\mathrm{KOH}$ mount irrespective of DNE findings. The observed findings indicate the variable presentation of CAM. The aggressiveness of CAM has changed, with normal nasal findings it has the potential to invade orbit, sinuses, and brain. Imaging plays an important role in making an early diagnosis. The reason attributed for this lack of enhancement is the angioinvasive nature of fungal hyphae which infiltrates the vessels supplying the mucosa ${ }^{5,6,7}$. In our study, it was found that black turbinate sign was present in the majority of the cases in MRI findings specific to sinonasal involvement of disease. With these findings in the imaging, even a negative DNE mandates a tissue diagnosis.

There is scarce literature among diagnostic utility of nasal endoscopy in relation to common clinical, microbiological, and radiological criteria in CAM.

Mohindra $\mathrm{S}$ et $\mathrm{al}^{8}$ reported in their study of 27 patients that the clinical suspicion, supplemented with radiological investigations aid in making a correct diagnosis. In our study, we found that even a negative endoscopy does not rule out the diagnosis of CAM. We would like to propose that all target group patients should be subjected to biopsy for $\mathrm{KOH}$ mount and imaging. Ferguson BJ et $\mathrm{al}^{9}$ found that the aggressive evaluation of patients with biopsy contributes to early detection of disease, which was in coherence to our study. We found that in many patients the disease pattern was sinus involvement before nasal involvement. Due to certain limitations of DNE, it is not able to diagnose sinus involvement in endoscopy, where imaging is paramount.

Gillespie MB et $\mathrm{al}^{10}$ showed the approach to diagnose the disease at an earlier stage while it is confined to the nasal cavity before the widespread extension to have a positive impact on survival.

Few authors have reported that turbinate sign is a radiological feature in MRI which can aid in earlier detection of the disease ${ }^{7}$. It is a moderately specific but significant finding in rhino-orbito-cerebral mucormycosis. In our study, it was found that a black turbinate sign in MRI is specific to sinonasal involvement of disease. To summarize, in the setting of COVID-19, an Otorhinolaryngologist must be vigilant at the imaging findings of CAM. One should have a low threshold for biopsy and imaging even in cases with normal endoscopy in target group patients to reduce false-negative results

\section{Limitations of the study}

This paper describes a single institution study, with a limited sample size. CEMRI could not be obtained for all patients because of financial and logistic constraints. However, this is likely representative of most 
high volume clinical centers across the developing world. Additional multi-institutional studies that include large sample size might be conducted to get more clear picture of the diagnostic modalities.

\section{Conclusion}

CEMRI is a valuable diagnostic test in suspected CAM and has high sensitivity and high accuracy compared to DNE. Although, DNE is a preferred and early diagnostic tool for the clinical assessment of CAM in resource constraint settings, a normal DNE does not rule out invasive fungal sinusitis. Imaging and biopsy should be considered in target group patients for early detection and better outcomes.

Disclosures: This manuscript is original and it, or any part of it, has not been previously published; nor is it under consideration for publication elsewhere. This research did not receive any specific grant from funding agencies in the public, commercial, or not-for-profit sectors. The authors declare that they have no conflicts of interest.

\section{References-}

[1] Yang W, Cao Q, Qin L, Wang X, Cheng Z, Pan A, et al. Clinical characteristics and imaging manifestations of the 2019 novel coronavirus disease (COVID-19): a multi-center study in Wenzhou city, Zhejiang, China. J Infect. 2020;80:388-93.

[2] Sen M, Lahane S, Lahane TP, Parekh R, Honavar SG. Mucor in a viral land: A tale of two pathogens. Indian J Ophthalmol. 2021;69:244-52.

[3] Honavar SG. Code Mucor: Guidelines for the Diagnosis, Staging and Management of Rhino-OrbitoCerebral Mucormycosis in the Setting of COVID-19. Indian J Ophthalmol. 2021;69:1361-5.

[4] Mathur S, Karimi A, Mafee MF. Acute optic nerve infarction demonstrated by diffusion-weighted imaging in a in a case of rhinocerebral mucormycosis. Am J Neuroradiol. 2007;28:489-90.

[5] Chae HK, Lim J, Lee SC, Kim KS. The Significance of "Black Turbinate Sign" on MRI. Ear Nose Throat J. 2020;99:395-6.

[6] Joshi AR, Muthe MM, Patankar SH, Athawale A, Achhapalia Y. CT and MRI Findings of Invasive Mucormycosis in the Setting of COVID-19: Experience From A Single Center in India. AJR Am J Roentgenol. 2021 Jun 23. doi:10.2214/AJR.21.26205.

[7] Safder S, Carpenter JS, Roberts TD, Bailey N. The "Black Turbinate" sign: An early MR imaging finding of nasal mucormycosis. AJNR Am J Neuroradiol. 2010;31:771-4.

[8] Mohindra S, Mohindra S, Gupta R, Bakshi J, Gupta SK. Rhinocerebral mucormycosis: the disease spectrum in 27 patients. Mycoses. 2007;50:290-6.

[9] Ferguson BJ. Mucormycosis of the nose and Paranasal sinuses. Otolaryngol Clin North Am. 2000;3:34965.

[10] Gillespie MB, O'Malley BW. An algorithmic Approach to the diagnosis and management of invasive fungal rhinosinusitis in the immunocompromised patient. Otolaryngol Clin North Am 2000; 33:323-34.

Tables

\begin{tabular}{llllll}
\hline Endoscopy findings & Endoscopy findings & Endoscopy findings & MRI findings & MRI findings & MRI 1 \\
\hline & Frequency & Percentage & & Frequency & Perce \\
Middle turbinate & 46 & $93.87 \%$ & Maxillary sinus & 54 & $90 \%$ \\
Inferior turbinate & 25 & $51.02 \%$ & Ethmoid sinus & 47 & $78.33 \%$ \\
Nasal septum & 4 & $8.16 \%$ & Sphenoid sinus & 35 & $58.33 \%$ \\
Superior turbinate & 3 & $6.12 \%$ & Frontal sinus & 25 & $41.66 \%$ \\
& & & Orbital extension & 12 & $20 \%$
\end{tabular}




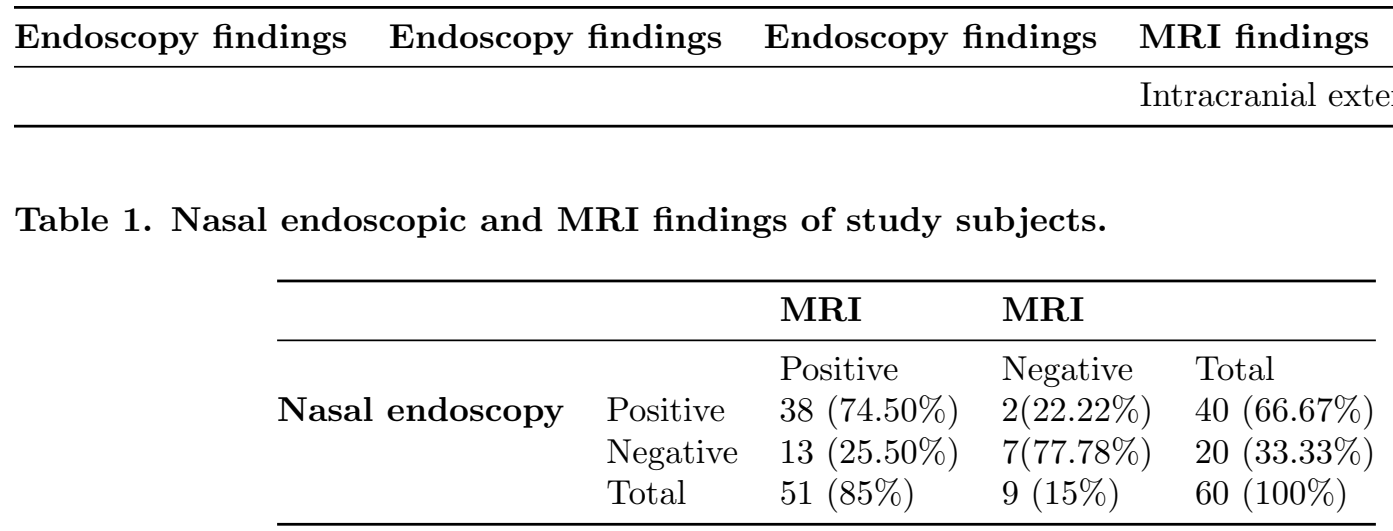

Table 2. Correlation between diagnostic nasal endoscopy and CEMRI in CAM patients $(\mathrm{n}=$ 60)Fig. 1. Clinical pictures demonstrating: (A) blackening of left cheek, left facial palsy, and involvement of $3^{\text {rd }}$ and $6^{\text {th }}$ cranial nerves; (B) palatal discoloration and eschar formation (C) complete ptosis of both eyes with necrosis and crusting (D) severe chemosis and complete ophthalmoplegia of right eye (E) diagnostic nasal endoscopy demonstrating black colored right middle turbinate; (B) black colored right inferior turbinate.Fig. 2. Contrast-enhanced magnetic resonance imaging of paranasal sinuses demonstrating: $(A, B)$ involvement of intraconal orbit and intracranial extension in axial planes; (C,D) involvement of maxillary and ethmoid sinus with heterogenous enhancement in coronal planes.
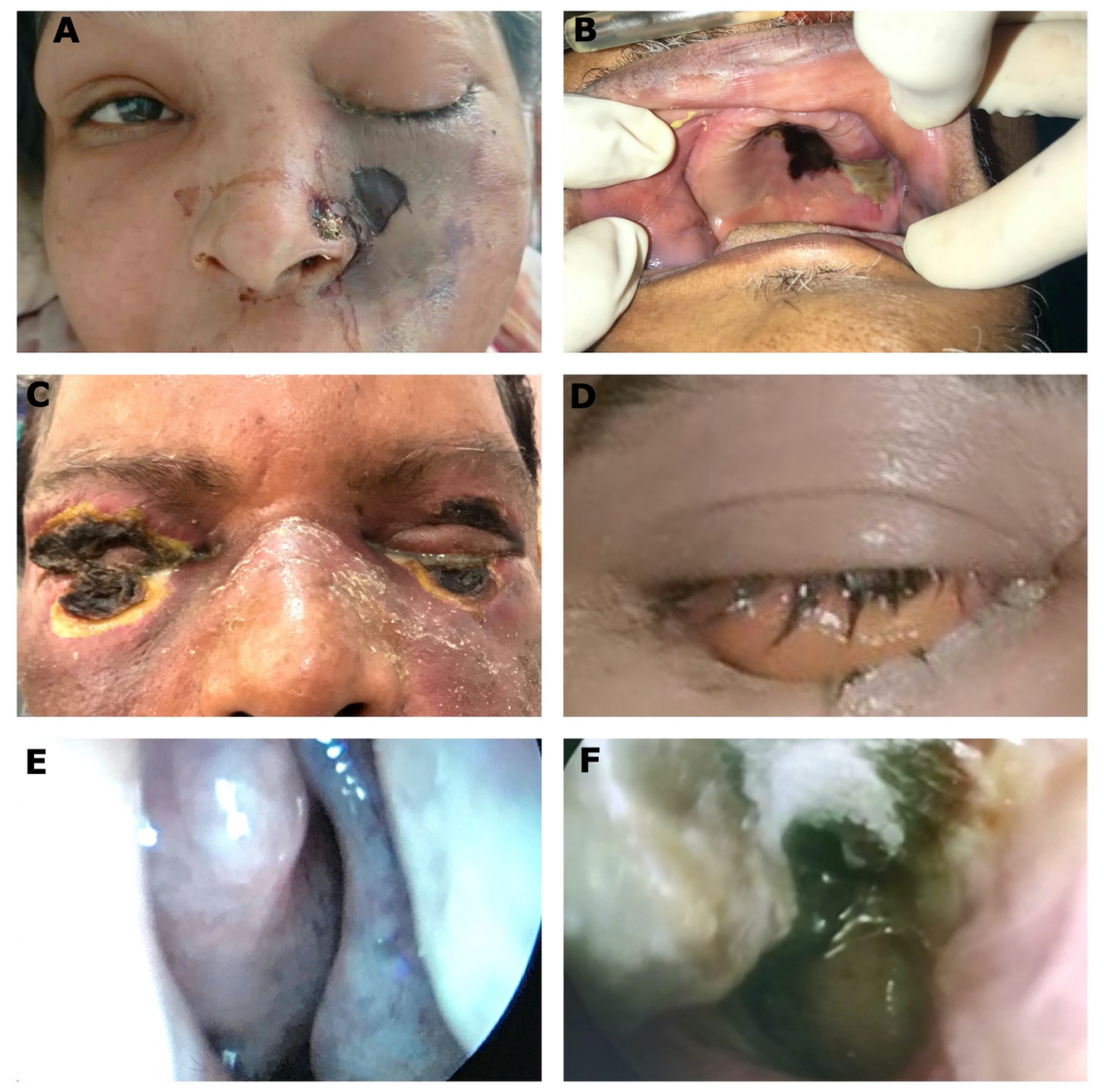

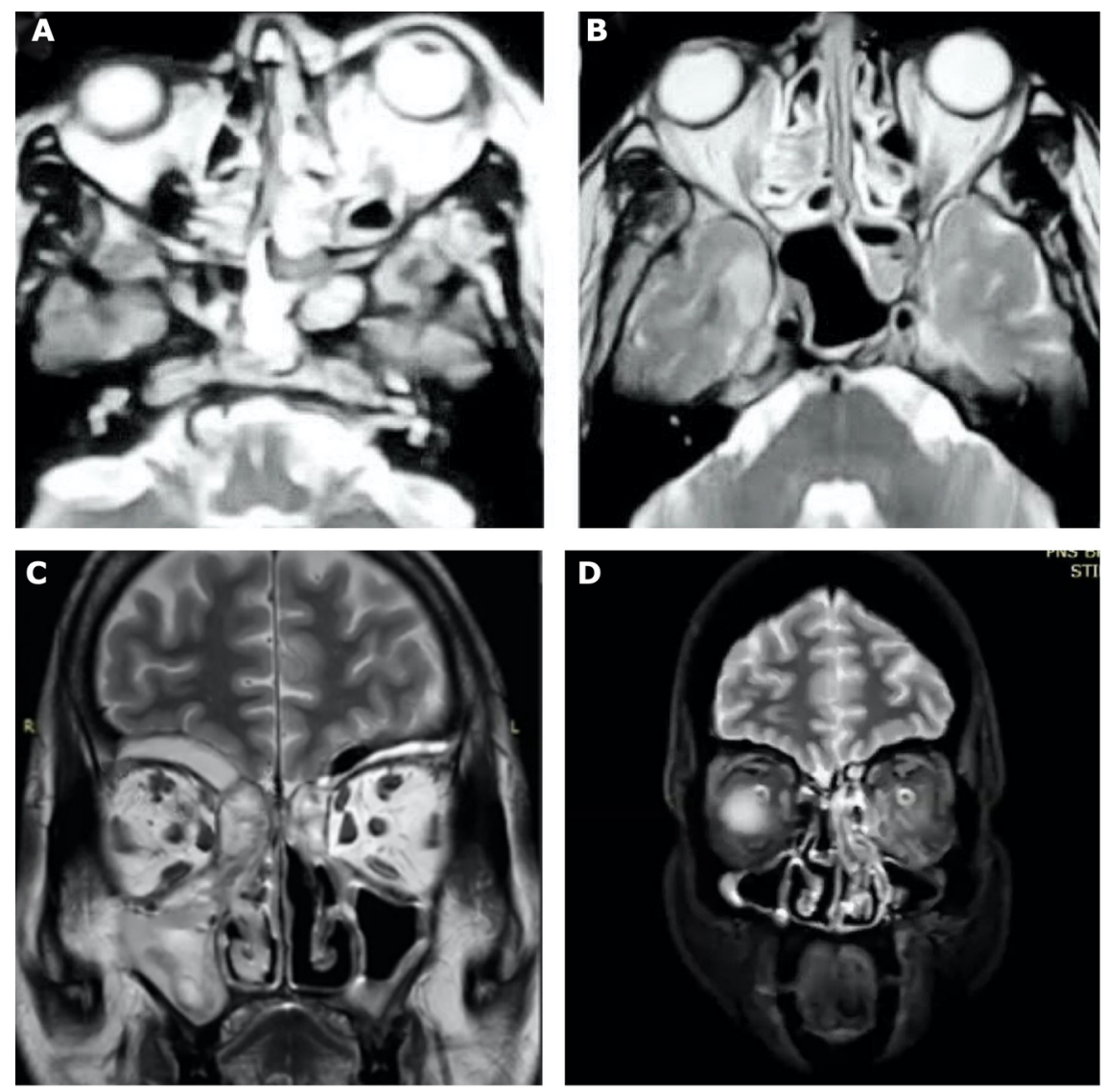